\title{
AVALIAÇÃO DA AÇÃO dO USO DE DROGAS NA SAÚDE BUCAL DE DEPENDENTES QUÍMICOS
}

\author{
Daiane Mayara Alves, Gisele Alborghetti Nai, José Luiz Santos Parizi \\ Universidade do Oeste Paulista - UNOESTE, Faculdade de Odontologia de Presidente Prudente - UNOESTE, SP. \\ Correspondência para: José Luiz Santos Parizi - parizi.patologia@unoeste.br
}

\section{RESUMO}

O uso de drogas de abuso é um problema de saúde publica. Uma das dificuldades para tratar os pacientes usuários de drogas é a carência de informações sobre o assunto voltado para a odontologia. Este estudo buscou avaliar as condições bucais de dependentes químicos em tratamento em Centros de Atenção Psicossocial (CAPS II AD). Foi realizada uma entrevista, exame da cavidade bucal, avaliação de sialometria e pH bucal em 60 pacientes atendidos por dois CAPS II $A D$ de duas cidades do interior do estado de São Paulo, de ambos os sexos e maiores de 18 anos. $A$ maioria (28\%) tinha idade entre 41 a 50 anos, eram do gênero masculino (85\%) e ensino fundamental incompleto (37\%). Entre os entrevistados, $98 \%$ relataram que já foram ao dentista. $22 \%$ não escovavam os dentes e $95 \%$ não faziam uso do fio dental. As substâncias químicas de abuso mais utilizadas foram: álcool (95\%), cigarro (85\%), maconha (48\%) e cocaína/crack/merla (40\%). A via de administração da droga mais citada foi a oral (51\%). Diagnosticou-se hipossalivação em $56 \%$ dos pacientes e $\mathrm{pH}$ ácido em $12 \%$. As principais alterações visíveis do periodonto foram retração gengival, alteração de cor, placa e cálculo simultaneamente (42\%), e a dental foi pigmentação exógena (15\%). Apenas $2 \%$ não possuíam nenhum dente permanente cariado, restaurado, perdido ou com extração indicada. Os pacientes avaliados apresentavam várias alterações bucais associadas ao uso de drogas de abuso (como hipossalivação, pH bucal ácido, pigmentação exógena), além de saúde bucal muito comprometida.

Palavras-chave: Transtornos relacionados ao uso de substâncias, doenças da boca, alcoolismo, hábito de fumar, cocaína.

\section{EVALUATION OF THE ACTION OF DRUG USE IN THE ORAL HEALTH OF ADDICTS}

\begin{abstract}
The use of drugs of abuse is a public health problem. One of the difficulties in treating drug users is the lack of information on the subject facing dentistry. This study aimed to assess the oral conditions of addicts in treatment in Centers for Psychosocial Care (CAPS II AD). We performed an interview, examination of the oral cavity, sialometry assessment and oral pH in 60 patients attending two CAPS II AD in two cities in the state of São Paulo, of both sexes and over 18 years old. The majority (28\%) were aged between 41 and 50 years, were male (85\%) and have elementary education (37\%). Among those interviewed, 98\% reported that they had been to the dentist. $22 \%$ did not brush their teeth and $95 \%$ did not flossing. The abuse drugs more used were: alcohol (95\%), cigarettes (85\%), marijuana (48\%) and cocaine / crack / merla (40\%). The oral route is the route of administration of the drug most frequently cited (51\%). Hyposalivation was diagnosed in $56 \%$ of patients and $12 \%$ had acidic $\mathrm{pH}$. The main visible changes in periodontal area were gingival retraction, color change, plaque and calculus simultaneously (42\%), and dental change was exogenous pigmentation (15\%). Only $2 \%$ had none decayed, restored, lost or extraction indicated in permanent teeth. All patients had multiple oral abnormalities associated with use of drugs of abuse (such as hyposalivation, oral acid pH, exogenous pigmentation), and oral health greatly compromised.
\end{abstract}

Key words: Substance-related disorders, mouth diseases, alcoholism, smoking, cocaine. 


\section{INTRODUÇÃO}

A dependência em drogas é uma doença complexa, caracterizada por uma busca compulsiva, algumas vezes incontrolável, sendo que seu uso persiste mesmo diante de consequências extremamente negativas. Estudos epidemiológicos sobre o consumo de droga apontam que este fenômeno tem ganhado um problema dimensional na Saúde Pública (BARROS; PILLON, 2006).

A Política Nacional de Saúde Mental, apoiada na lei 10.216 de 06 de abril de 2001, buscou consolidar um modelo de atenção à saúde mental aberto e de base comunitária (BRASIL, 2001). Os Centros de Atenção Psicossocial (CAPS) fazem parte da nova política em saúde mental no Brasil (PILLON; MARTINS, 2008).

Os CAPS II ad (serviço de atenção psicossocial para atendimento de pacientes com transtornos decorrentes do uso e dependência prejudicial de substâncias psicoativas) são uma rede estratégica de serviços extra-hospitalares que constitui em serviço ambulatorial de atenção diária (BRASIL, 2002).

Cada substância de abuso, lícita ou ilícita, tem seu poder devastador sobre os tecidos bucais (COLODEL et al., 2008). A ação local das drogas na cavidade bucal apresenta efeitos deletérios, resultando em nível elevado de CPOD (dentes cariados, perdidos, obturados e com extração indicada), gengivite, halitose, estomatite, bruxismo, desgaste dentais, queilite angular, entre outros (GUPTA et al., 2012). As complicações odontológicas específicas do uso de drogas incluem também cárie excessiva e distúrbios periodontais de rápida evolução, que podem estar relacionados com deficiências nutricionais e negligência da higiene pessoal (PÉRET; BONATO, 2008; GUPTA et al., 2012).

Há uma carência de informações sobre o tema voltado para a Odontologia, para subsidiar a conduta dos profissionais frente a um dos maiores problemas de saúde pública, que afeta tanto diretamente quanto indiretamente a qualidade de vida dos usuários e seus familiares.

O objetivo deste estudo foi avaliar as condições bucais de dependentes químicos em tratamento em Centros de Atenção Psicossocial (CAPS) II ad.

\section{MÉTODOS}

Este estudo foi aprovado pelo Comitê de Ética em Pesquisa da Universidade do Oeste Paulista (CEP/UNOESTE - Processo $\mathrm{n}^{\circ}$ 869/11).

Participaram da pesquisa 60 pacientes, de ambos os sexos e maiores de 18 anos. O critério de inclusão na pesquisa foi ser paciente que se encontrava em tratamento no Centro de Atenção Psicossocial - CAPS II ad - e que concordou 
com a participação no estudo mediante assinatura do termo de consentimento livre e esclarecido (TCLE).

Primeiramente, foi realizada uma entrevista com o auxílio de um questionário estruturado, previamente validado com $10 \%$ da amostra atendida, e posterior exame físico, intra e extrabucal nos pacientes atendidos por dois CAPS II ad de duas cidades do interior do estado de São Paulo.

A coleta dos dados foi realizada no próprio CAPS II ad. Os pacientes foram convidados a participar da pesquisa no momento em que compareceram ao CAPS para a sua consulta de rotina.

$\mathrm{Na}$ entrevista foram coletados dados sobre a identificação do paciente, caracterização socioeconômica, escolaridade, tratamento médico atual e medicações em uso, tratamento odontológico atual, tempo da última consulta ao dentista, motivação do paciente a buscar o atendimento odontológico, uso diário de escova dental e fio dental (quantas vezes ao dia), principais drogas de uso, tempo e frequência de uso, via de administração da substância de abuso e tempo de abstinência.

Os pacientes foram examinados individualmente, por dois pesquisadores simultaneamente (DMA e JLSP), sentados em uma cadeira comum, sob iluminação natural, com o auxílio de materiais previamente esterilizados, como jogo clínico (espelho, sonda e pinça), abaixador de língua e gazes. A definição do diagnóstico clínico do paciente foi dada em conjunto pelos dois examinadores, após discussão do caso.

O exame extrabucal constituiu-se de palpação da cadeia linfática (parotídea, submandibular, sub-mentoniana e cervical) e da verificação de assimetrias faciais. O exame intrabucal contou com avaliação dos tecidos moles (semimucosa labial, mucosa da bochecha e fundo de fórnix, ventre, assoalho e bordas da língua, e pilares posteriores da garganta), exame gengival (avaliação da presença de retrações, bolsas gengivais, alterações de cor e processo inflamatório), avaliação do palato duro e mole e avaliação dental com determinação do índice CPOD (dentes cariados, perdidos, obturados e com extração indicada) e presença de abrasão, erosão, atrição ou pigmentações exógenas.

O teste com azul de metileno, teste de $\mathrm{pH}$ salivar e teste de fluxo salivar também foram realizados.

O teste com azul de metileno foi aplicado somente em lesões brancas, para determinação da necessidade da realização de biópsia ou não.

Para a determinação da sialometria total (medição do fluxo salivar), foi utilizado um estímulo mecânico (garrote de mangueira de látex), tubos de ensaios calibrados em pelo menos $0,1 \mathrm{ml}$ e cronômetro. Após a introdução da borracha na boca e o início de 
sua mastigação, começou-se a contagem com o cronômetro de um tempo de seis minutos. A saliva produzida no primeiro minuto foi desprezada e a produzida nos cinco minutos seguintes foi coletada, a cada intervalo de um minuto, em um recipiente graduado. Após esse procedimento o operador anotou o volume de saliva sendo que a taxa do fluxo salivar foi expressa em milímetros por minuto ( $\mathrm{ml} / \mathrm{min})$. Os valores foram analisados da seguinte forma: fluxo normal de 1,0 a 3,0 $\mathrm{ml} / \mathrm{min}$, baixo fluxo de 0,7 a $1,0 \mathrm{ml} / \mathrm{min}$ e hiposalivação menos de $0,7 \mathrm{ml} / \mathrm{min}$ (SILVA et al., 2007; BRETAS et al., 2008).

Imediatamente após a coleta da saliva, foi colocada uma tira indicadora de $\mathrm{pH}$, com variação entre pH 0,0 a 14,0 (MachereyNagel GmbH \& Co. KG, Alemanha) no interior do dispositivo coletor para a determinação do $\mathrm{pH}$ salivar. A determinação do $\mathrm{pH}$ salivar com o emprego de tiras indicadoras de $\mathrm{pH}$ é feita pelo método colorimétrico (medição com as tiras indicadoras de $\mathrm{pH}$ ), que utiliza uma escala própria para leitura. Dez minutos depois, o pH de repouso foi identificado pela coloração da fita indicadora de pH conforme indicação do fabricante, considerando como parâmetro de normalidade $\mathrm{o}$ pH 7,0 (SERRATINE; SILVA, 2009).

Os pacientes, que apresentaram alterações bucais e que necessitaram de tratamento, foram encaminhados para a Unidade Básica de Saúde (UBS) do município ao qual o CAPS II ad pertencia.

Para a análise estatística utilizou-se o teste do qui-quadrado. 0 nível de significância utilizado foi de $5 \%$. Os testes estatísticos foram realizados com o pacote SPSS - Statistical Package for the Social Sciences v.15.0.

\section{RESULTADOS}

Dos 60 entrevistados, a maioria tinha entre 41 a 50 anos (28\%) (Figura 1), era do sexo masculino (85\%) e solteiro (45\%).

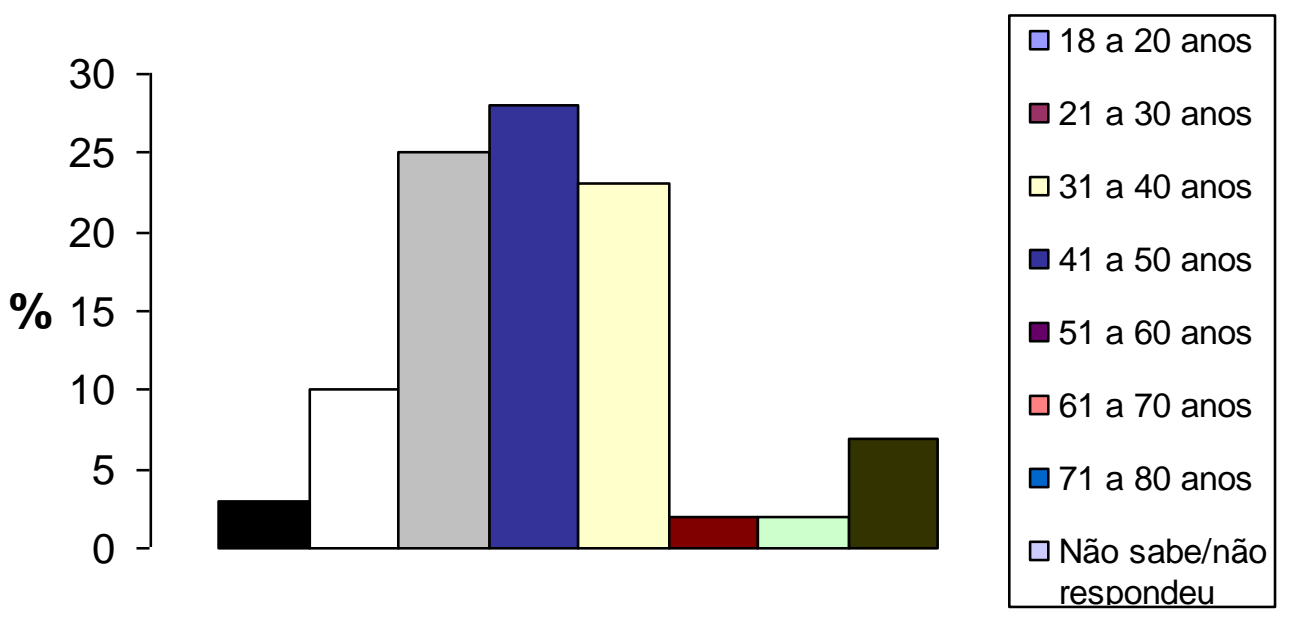

Figura 1. Faixa etária dos pacientes avaliados $(n=60)$. 
Em relação ao perfil socioeconômico, $73 \%$ dos entrevistados não estava trabalhando no momento, e destes $12 \%$ recebiam auxílio-doença devido a estar em tratamento no CAPS AD. Quanto à renda familiar, $73 \%$ relataram ser entre $R \$ 501$ a $\mathrm{R} \$ 1500,00$ e, em relação à escolaridade, a maioria (37\%) tinha o ensino fundamental incompleto (Figura 2).

\section{tratamento no CAPS AD. Quanto à renda}
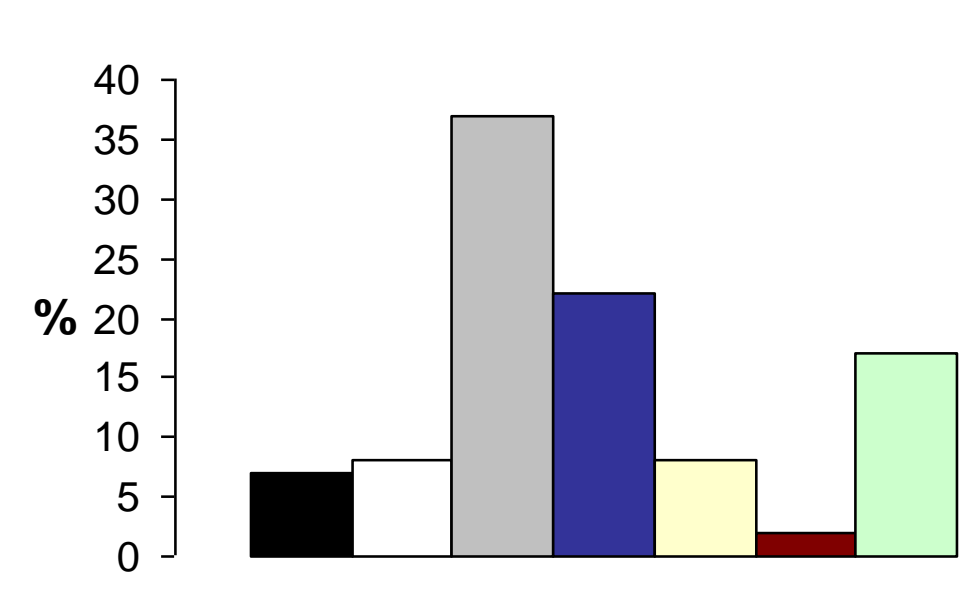

\begin{tabular}{|c|}
\hline$\square$ Não \\
alfabetizado \\
$\square$ Alfabetizado \\
$\square$ Fundamental \\
incompleto \\
$\square$ Fundamental \\
completo \\
$\square$ médio \\
imcompleto \\
$\square$ Médio \\
completo \\
$\square$ Não sabe/não \\
respondeu \\
\hline
\end{tabular}

Figura 2. Nível de escolaridade dos pacientes avaliados $(n=60)$.

Na Tabela 1, encontram-se dispostas

as substâncias químicas utilizadas pelos pacientes avaliados. 
Tabela 1. Prevalência das drogas de abuso utilizadas pelos pacientes avaliados ( $n=60)$.

\begin{tabular}{lcccc}
\hline \multirow{2}{*}{ Substância química utilizada } & \multicolumn{3}{c}{ Sim } & \multicolumn{2}{c}{ Não } \\
\cline { 2 - 5 } & $\mathbf{n}$ & $\mathbf{\%}$ & $\mathbf{n}$ & $\%$ \\
Álcool & 57 & 95 & 3 & 5 \\
Cigarro & 51 & 85 & 9 & 15 \\
Maconha & 29 & 48 & 31 & 52 \\
Cafeína & 26 & 43 & 34 & 57 \\
Cocaína/Crack/Merla & 24 & 40 & 36 & 60 \\
Tranquilizantes/ansiolíticos & 16 & 27 & 44 & 73 \\
Calmantes/sedativos & 12 & 20 & 48 & 80 \\
Solventes/inalantes & 11 & 18 & 49 & 82 \\
Anfetamina & 5 & 8 & 55 & 92 \\
Plantas alucinógenas & 5 & 8 & 55 & 92 \\
Ópio & 1 & 2 & 59 & 98 \\
Ópio & 1 & 2 & 59 & 98 \\
Anabolizantes & 1 & 2 & 59 & 98 \\
Anticolinérgicos & 1 & 2 & 59 & 98 \\
Oxi & 1 & 2 & 59 & 98 \\
LSD & 0 & 0 & 60 & 100 \\
\hline
\end{tabular}

Aos pacientes que relataram utilizar a quantidade, e os valores estão descritos na Figura 3.

cigarro foram questionados quanto ao tipo e

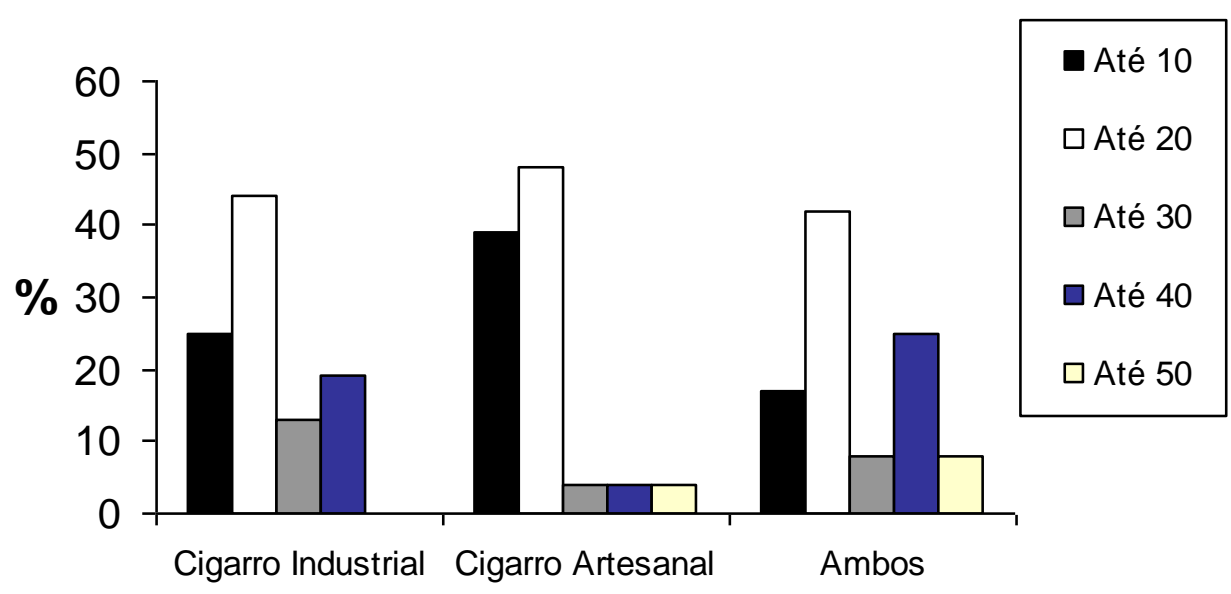

Figura 3. Quantidade de cigarro utilizada por dia pelos pacientes avaliados em relação ao tipo $(n=60)$.

Dos pacientes avaliados, $75 \%$ continuam fazendo uso de algum tipo de 
droga e a principal via de administração foi a via oral (52\%), seguida de oral/inalatória (40\%).
As Figuras 4 e 5 mostram o número de anos que os pacientes faziam uso das drogas de abuso e a frequência de uso, respectivamente.

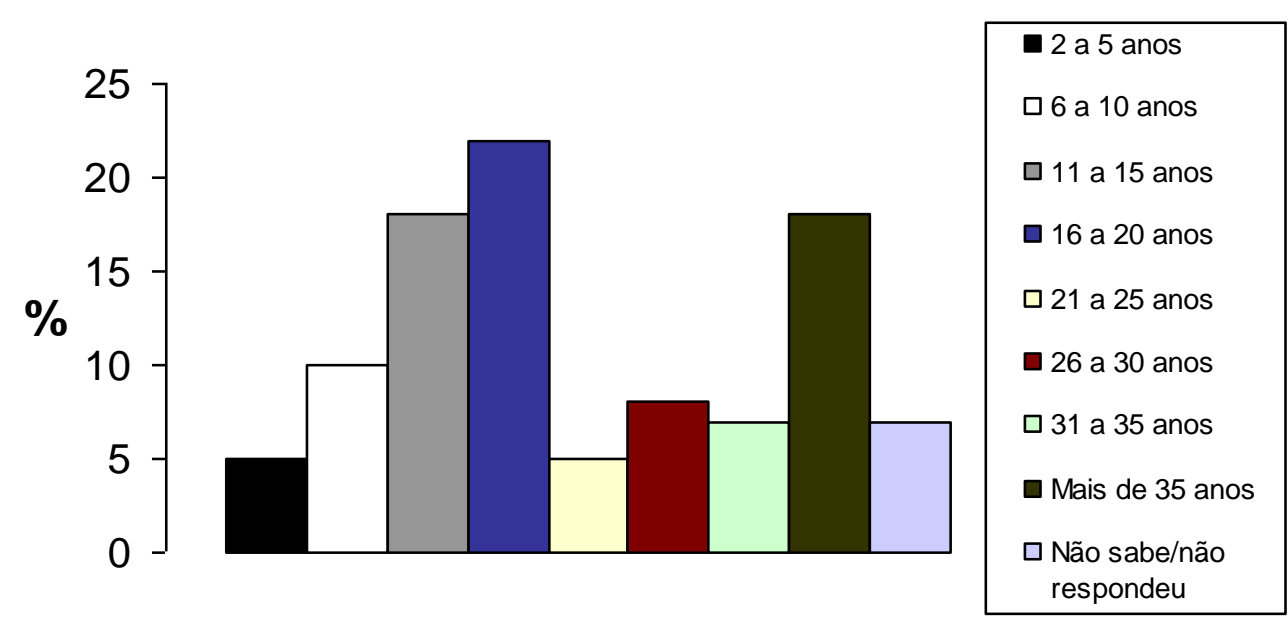

Figura 4. Tempo de uso das drogas de abuso pelos pacientes avaliados $(n=60)$.

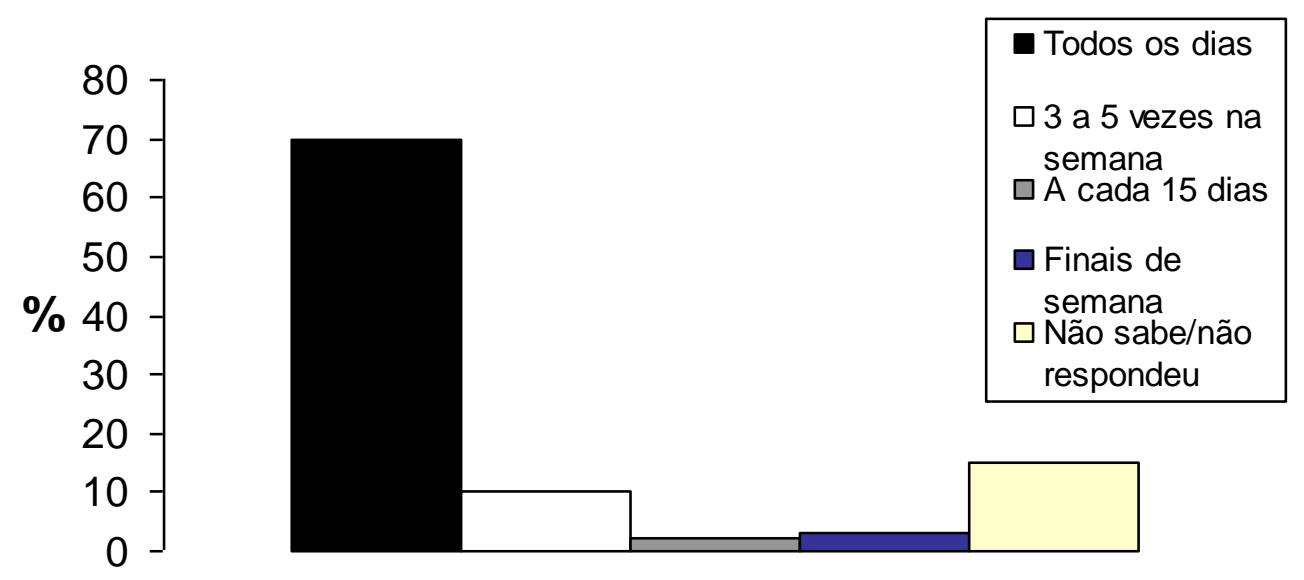

Figura 5. Frequência do uso de drogas pelos pacientes avaliados $(n=60)$.

A maioria (87\%) relatou estar antipsicótico, tranquilizantes e adesivo para realizando algum tipo de tratamento médico no momento, e $93 \%$ estavam fazendo uso de parar de fumar.

Com relação à consulta odontológica, algum medicamento de uso contínuo, sendo 98\% dos pacientes responderam que alguma os principais citados: antidepressivos, vez na vida já haviam ido ao dentista, e 7\% antiepiléticos, ansiolíticos, sedativos, destes estavam realizando algum tratamento odontológico no momento. A última consulta 
odontológica foi realizada pela maioria (37\%) entre dois a três anos (Figura 6).

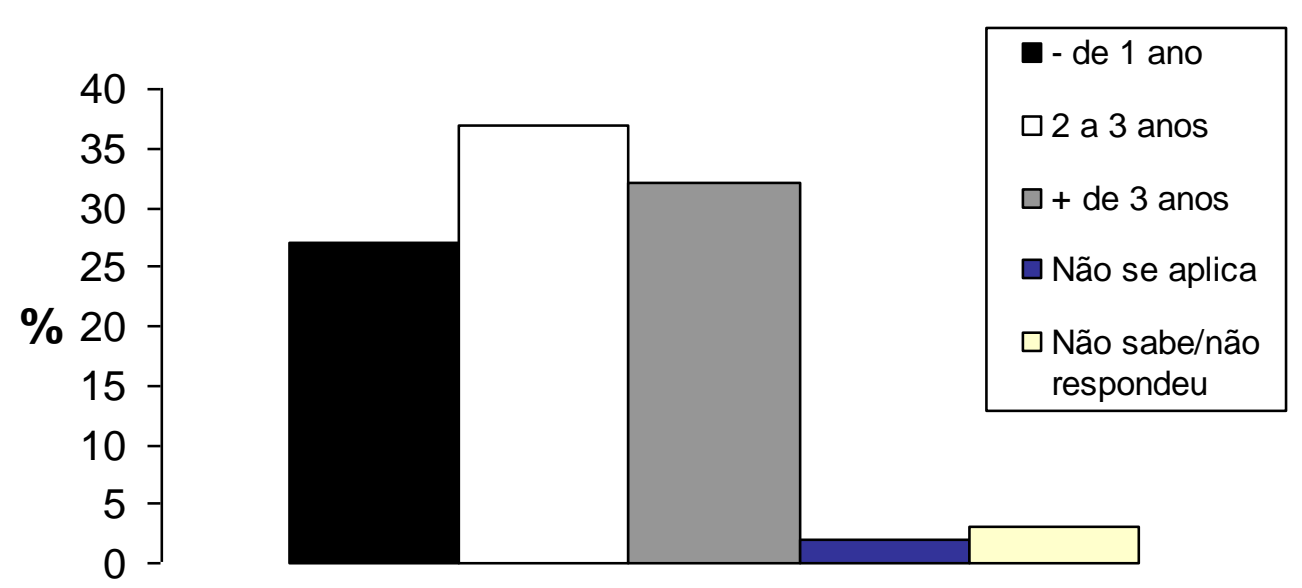

Figura 6. Tempo da última consulta odontológica dos pacientes avaliados ( $n=60)$.

A maioria dos pacientes $(90 \%)$ acreditava que necessitava de algum tipo de tratamento odontológico no momento e as principais respostas para o tipo de tratamento necessário foram: necessidade de prótese, extração, restauração, avaliação de fratura dentária, dores, sensibilidade dental, limpeza dos dentes, prevenção e correção ortodôntica.

No que diz respeito aos hábitos de higiene bucal, a maioria dos avaliados (78\%) relatou realizar escovação diária, predominantemente duas vezes ao dia, e apenas $5 \%$ relataram utilizar o fio dental.
Quanto á analise do fluxo salivar realizada nos pacientes, $56 \%$ apresentaram hipossalivação $(p<0,05)$. Em relação á análise do $\mathrm{pH}$ salivar, observou-se que $12 \%$ possuíam $\mathrm{pH}$ ácido $(\mathrm{p}<0,05)$.

Apenas um dos pesquisados apresentou gânglio infartado e dois apresentaram assimetria facial.

Na Tabela 2 estão apresentados os principais achados na avaliação bucal dos pacientes. 
Tabela 2. Principais achados da avaliação bucal realizada nos pacientes ( $n=60) . p<0,05$

\begin{tabular}{|c|c|c|}
\hline Avaliação bucal & $\mathbf{n}$ & $\%$ \\
\hline \multicolumn{3}{|l|}{ Alterações de partes moles } \\
\hline Nada digno de nota & 40 & 65 \\
\hline Hiperceratose somente & 4 & 6,5 \\
\hline Varicosidade lingual somente & 5 & 8 \\
\hline Nódulo somente & 1 & 2 \\
\hline Fístula somente & 2 & 3 \\
\hline Leucoplasia somente & 1 & 2 \\
\hline Varicosidade lingual e fístula & 1 & 2 \\
\hline Leucoplasia e hiperceratose & 1 & 2 \\
\hline Fibrose gengival somente & 1 & 2 \\
\hline Leucoplasia e lesão negra do palato & 1 & 2 \\
\hline Lesão negra do palato somente & 1 & 2 \\
\hline Eritroplasia no palato somente & 1 & 2 \\
\hline Hiperplasia fibrosa inflamatória na mucosa jugal & 1 & 2 \\
\hline \multicolumn{3}{|l|}{ Alteração visível do periodonto } \\
\hline Nada digno de nota & 20 & 33 \\
\hline Retração gengival somente & 2 & 3 \\
\hline Placa e cálculo & 5 & 8 \\
\hline Retração gengival e placa & 4 & 7 \\
\hline Retração gengival, alteração de cor, placa e cálculo & 25 & 42 \\
\hline Alteração de cor e placa & 2 & 3 \\
\hline Alteração de cor, placa e cálculo & 1 & 2 \\
\hline Gengivite e placa & 1 & 2 \\
\hline \multicolumn{3}{|l|}{ Avaliação da arcada dentária } \\
\hline Nada digno de nota & 27 & 45 \\
\hline Pigmentação exógena e atrição & 9 & 15 \\
\hline Atrição e fratura dental & 2 & 3 \\
\hline Atrição somente & 3 & 5 \\
\hline Fratura dental somente & 1 & 2 \\
\hline Fratura dental e pigmentação exógena & 2 & 3 \\
\hline Pigmentação exógena somente & 9 & 15 \\
\hline Abrasão somente & 1 & 2 \\
\hline Pigmentação exógena, atrição e fratura dental & 2 & 3 \\
\hline Pigmentação exógena e abrasão & 4 & 7 \\
\hline \multicolumn{3}{|l|}{ Índice CPOD } \\
\hline 0 & 1 & 2 \\
\hline 1 a 5 & 4 & 7 \\
\hline 6 a 10 & 11 & 18 \\
\hline 11 a 15 & 7 & 12 \\
\hline 16 a 20 & 14 & 23 \\
\hline 21 a 25 & 7 & 12 \\
\hline 26 a 32 & 16 & 27 \\
\hline
\end{tabular}


Quanto ao uso de prótese em um ou ambos maxilares, foi detectado que $37 \%$ usavam algum tipo de prótese e 70\% tinham necessidade de uso ou troca. Em relação ao tipo de prótese, a maioria (45\%) usava prótese total, e $40 \%$ necessitavam de prótese total ou parcial (Figura 7). Com relação ao arco, a maioria dos pacientes com prótese (73\%) utilizava no arco superior, e $79 \%$ necessitavam em ambas arcadas (Figura 8).

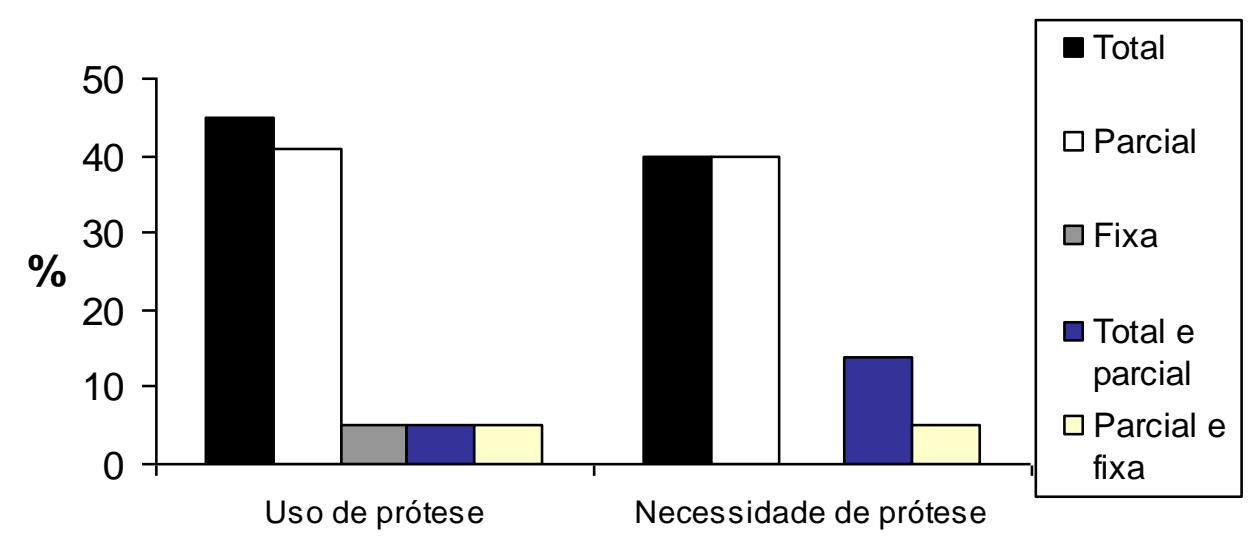

Figura 7. Tipo de prótese utilizada e necessária aos pacientes avaliados ( $n=60)$.

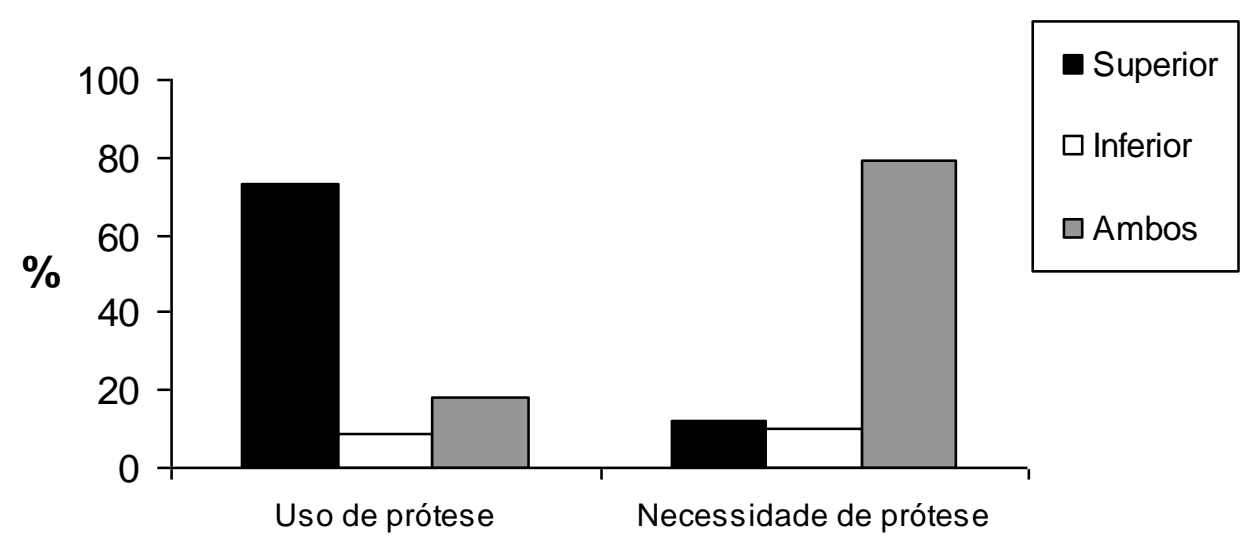

Figura 8. Arco de utilização e de necessidade de prótese pelos pacientes avaliados ( $n=60)$.

O teste com azul de metileno foi aplicado somente em três pacientes que apresentavam área de leucoplasia, o qual resultou negativo, não necessitando encaminhamento para biópsia.

\section{DISCUSSÃO}

Neste estudo, a maioria dos pacientes tinha entre 31 e 60 anos (76\%) e eram do gênero masculino (85\%), mostrando que o vício em drogas de abuso atinge um grande número de indivíduos na fase ativa da vida laboral. Há grande abandono do emprego, 
como também observado neste estudo, onde $73 \%$ dos entrevistados não trabalhavam, e da parte educacional, onde se observou que $59 \%$ tinham apenas o ensino fundamental. A baixa renda familiar (maioria estava em 500 e 1500 reais) e a baixa escolaridade, além de poder contribuir para que 0 paciente continue com o abuso de drogas, também pode contribuir para que o mesmo não procure assistência médica e odontológica quando necessário ou de forma preventiva, além deste não se preocupar com os cuidados pessoais. Na presente pesquisa, isto pode ser observado com respeito aos hábitos de higiene bucal, onde embora a maioria realizava a escovação diariamente (78\%), predominantemente duas vezes ao dia apenas, e somente $5 \%$ usavam fio dental.

As drogas de abuso podem ser introduzidas no organismo de várias maneiras, ou seja, podem ser inaladas, comidas ou bebidas, fumadas, aspiradas ou injetadas e a via de administração influenciará em seus efeitos (BARROS; PILLON, 2006). Neste estudo, a principal via de administração da droga foi oral (52\%), seguida de oral/inalatória (40\%), o que representa um impacto direto destas substâncias na cavidade oral.

É imprescindível conhecer os tipos de tóxicos utilizados, pois as drogas geralmente modificam o comportamento do paciente, como as funções básicas de alimentação e sono, provocando uma queda de resistência, e de higiene (GUPTA et al, 2012) podendo favorecer condições de língua sulcada ou pilosa principalmente em pacientes tabagistas (WASZKIEEWICZ et al., 2012a; 2012b) e podem ainda modificar a densidade da saliva, como por exemplo, esta pode apresentar-se viscosa, no caso do uso de maconha (SCHULZ-KATTERBACH et al., 2009) e da xerostomia provocada por certos tranquilizantes (D'AMORE et al., 2011). No presente estudo, as drogas mais utilizadas pelos entrevistados foram álcool (95\%), cigarro (85\%), maconha (48\%) e cocaína/crack/merla (40\%), as quais estão muito relacionadas a danos à saúde bucal.

Foi observado que os pacientes avaliados faziam uso por tempo prolongado das drogas, a maioria por 11 a 20 anos (40\%), e diariamente (70\%). Além disso, a maioria (75\%) continuava fazendo uso de drogas de abuso mesmo estando em tratamento. O uso prolongado e diário de droga pode ter um impacto muito grande sobre a saúde bucal, principalmente por que a principal via de administração das drogas é a oral ou inalatória.

Em relação ao uso de cigarro, a maioria dos entrevistados fumava até 20 cigarros por dia, independente do tipo (industrial ou artesanal). Pessoas que fazem o uso intenso 
e constante de cigarro têm mais chances de desenvolver câncer de boca e podem apresentar irritação crônica na mucosa do palato, denominada palatite nicotínica, e ainda leucoplasia, ambas são lesões brancas que podem ser consideradas cancerizáveis (ROSA et al., 2009; LOPES et al., 2012). O tabaco interfere nos processos químicos que acontecem na boca, como diminuição de imunoglobulina A na saliva contribuindo para pior estado periodontal (WASZKIEEWICZ et al., 2012b), além de acarretar em excesso de cálculo dental, deixar os dentes amarelos ou enegrecidos, acelerar a deterioração da arcada dentária, contribuir para o surgimento de cáries e aumentar o risco do fumante perder os dentes em $50 \%$ mais do que os não fumantes (OLIVEIRA, 2009). Estas alterações também foram observadas neste estudo, sendo que as mais encontradas no exame da cavidade bucal foram hiperceratose, presença de cálculos, retração gengival e pigmentação exógena nos dentes e na gengiva, além de grande perda dentária.

O uso de tabaco para mascar que é o tabaco in natura ou uma mistura de tabaco, traz significativos riscos para a saúde. Na boca é causa de retração e inflamação das gengivas, cáries e queda dos dentes e ainda aumento do risco de câncer de boca e leucoplasia (BRASIL, 2006). Outra forma de utilização do tabaco é o cigarro de palha.
Alguns estudos apontam para o fato de que fumantes de cigarro de palha apresentam níveis maiores de dependência à nicotina e risco aumentado de desenvolver doenças relacionadas ao tabaco (BRASIL, 2006). Neste estudo, mais de $60 \%$ fazia uso de cigarro de palha (associado ou não ao uso de cigarro industrial), mostrando que é importante que o paciente seja questionado quanto ao tipo de cigarro em uso, pois as consequências para saúde orgânica e bucal são distintas daquelas provocadas pelo cigarro industrializado.

O tratamento antitabágico pode trazer algumas reações adversas na cavidade bucal (ROSA et al., 2009). Os pacientes que estão sobre tratamento terapêutico para parar de fumar podem apresentar certas reações adversas na cavidade bucal como é o caso da goma de mascar ou tablete Nicorette que pode levar a um aumento da salivação, dor bucal, dor muscular na mandíbula e aftas e em alguns casos, a goma ou tablete pode também aderir à prótese, danificando-a (STEAD et al., 2008). Há ainda a terapia não nicotínica com o uso de Nortriptilina, que é um medicamento indicado em caso de depressão e que pode levar a xerostomia e raramente, a adenite sublingual associada (STEAD et al., 2008). Há também o cloridrato de bupropiona (Zyban ${ }^{\oplus}$ ), um medicamento que reduz a vontade de fumar de pessoas 
com dependência à nicotina. Seu uso pode trazer algumas reações adversas como xerostomia, vômito, alteração de paladar, e reações alérgicas, além de inchaço de lábios ou língua (STEAD et al., 2008). Deixar de fumar, ainda pode gerar um estresse psicológico aliado à redução da hiperqueratinização do epitélio oral podendo assim gerar aftas (ROSA et al., 2009). Neste estudo, apenas alguns pacientes faziam uso de adesivos de nicotina e nenhum fazia uso de goma de mascar com nicotina, sendo assim não se pode relacionar as alterações observadas na cavidade bucal destes indivíduos com o tratamento antitabágico.

Os indivíduos usuários de drogas apresentam uma alta prevalência de lesões cancerizáveis bucais quando comparados com a população em geral, tais como, queilite actínica, leucoplasia bucal, eritroplasia e líquen plano (FERNANDES et al., 2008; LOPES et al, 2012). Neste estudo, foram observados apenas três casos de leucoplasia e um de eritroplasia, mostrando que na população estudada não houve aumento da prevalência de lesões cancerizáveis, como também foi observado em outro estudo com pacientes de Centros de Reabilitação (O'SULLIVAN, 2011).

$\mathrm{O}$ etilismo frequentemente resulta em graves consequências, incluindo o aumento do risco de desenvolvimento de doença periodontal e de efeitos adversos na execução do tratamento da doença (PÉRET; BONATO, 2008). É um dos agentes químicos relacionados ao desenvolvimento de neoplasias malignas bucais (REIS et al., 2002; WASZKIEWICZ et al., 2012a). Quando o nível de alcoolemia é alto podem ocorrer náuseas e vômitos, que se forem constantes, podem favorecer erosão dental e cárie dental resultantes de um ambiente bucal cronicamente ácido (TRAEBERT; MOREIRA, 2001). Neste estudo, $67 \%$ dos pacientes apresentavam doença periodontal e $12 \%$ apresentaram $\mathrm{pH}$ ácido bucal, dados estes relacionados estatisticamente ao uso de droga e que pode parcialmente ser explicado pelo descrito acima.

A saliva é imprescindível para a manutenção da saúde dos dentes e mucosa bucal, pois afeta os dentes e a microbiota de várias maneiras, como por exemplo, uma diminuição do fluxo salivar (xerostomia) e baixa capacidade tampão, podem levar a baixa eliminação de microorganismos e restos alimentares, além de poder prejudicar na neutralização de ácidos, reduzindo assim a remineralização do elemento dental, podendo implicar num alto risco de cárie (WASZKIEWICZ et al., 2011; AHMADIMOTAMAYEL et al., 2012). A xerostomia e a hipossalivação devem ser consideradas não só um problema dental, mas também médico 
(NEDERFORS, 2000), pois está associada também a fisiologia esofágica, a digestão e citoproteção gástrica (WASZKIEWICZ et al., 2011) e pode ser causada por uso de medicações (THOMSON et al., 2006) e abuso de drogas como álcool (WASZKIEWICZ et al., 2011), maconha (SCHULZ-KATTERBACH et al., 2009) e metanfetaminas (HAMAMOTO; RHODUS, 2009; RAVENEL et al., 2012), por exemplo. Neste trabalho, 56\% apresentavam hipossalivação, a qual esteve relacionada ao uso de drogas $(p<0,05)$, o que pode ter favorecido o alto índice CPOD encontrado na maioria dos pacientes.

As principais substâncias químicas presentes nos solventes ou inalantes são os hidrocarbonetos como, por exemplo, o tolueno, xilol, acetona, acetato de etila e estes causam irritação das mucosas do nariz e da boca (RIDENOUR et al., 2007). No presente estudo, $18 \%$ dos avaliados faziam uso deste tipo de substância, o que também contribuiu para as alterações bucais observadas.

Os opiáceos formam um grupo de substâncias naturais ou sintéticas, derivadas do Ópio (D’AMORE et al., 2011). Além dos opióides poderem deprimir o sistema imunológico através de sua interação com os receptores dos opióides nos linfócitos $\mathrm{T}$ e leucócitos, ainda podem causar xerostomia e consequentemente aumentar o risco de cárie dental (D'AMORE et al., 2011). Neste estudo, somente um paciente fazia uso de opiáceos, o que pouco contribui para incidência de hipossalivação e perda dentária encontrada.

A denominação anfetamina é utilizada para designar todo um grupo de substâncias como: fenproporex, metilfenidato, manzidol, metanfetamina e dietilpropiona. Seu uso clínico mais comum é como moderador de apetite e no tratamento de transtorno de déficit de atenção/hiperatividade (RAVENEL et al., 2012). Há vários efeitos nocivos da metanfetamina sobre a saúde bucal, tais como pobre higiene oral, xerostomia, cáries exuberantes ("meth mouth"), desgaste dentário excessivo, perda dentária, presença de cálculos e diminuição de pH bucal (HENG et al., 2008; HAMAMOTO; RODHUS, 2009; NAIDOO; SMIT, 2011; RAVENESL et al., 2012). Outro tipo de anfetamina, porém de uso ilícito, é a metilenodioximetanfetamina (MDMA) ou Ecstasy (BRAND et al, 2008). Seus efeitos indesejáveis de interesse odontológico são principalmente bruxismo, mais acentuado nos dentes posteriores e que pode persistir após o uso da droga, espasmo maxilar, erosão dental, ressecamento da boca, perda de apetite e crises bulímicas (BRAND et al., 2008). Também pode causar disfunção do sistema imunológico, sendo esse quadro agravado quando há associação com álcool (BRAND et al., 2008). Neste 
estudo, $8 \%$ dos avaliados faziam uso de anfetaminas, o que pode ter contribuído também para a alta incidência de hipossalivação e diminuição do pH salivar dos pacientes. Muitos deles faziam uso concomitante de álcool, o que pode potencializar os efeitos danosos das anfetaminas sobre a saúde bucal dos pacientes avaliados.

A cocaína é uma substância natural extraída de uma planta existente na América do Sul, popularmente conhecida como Coca (Erythroxylon coca) (MALONEY, 2010). Pode ser consumida na forma de pó (cloridrato de cocaína), que pode ser aspirado ou dissolvido em água e injetada na corrente sanguínea, ou sob a forma de uma base, o crack, que é fumado (MALONEY, 2010; WOYCEICHOSKI et al., 2007). Existe ainda a pasta de coca, um produto menos purificado, que também pode ser fumado, conhecido como merla (SILVA JUNIOR et al., 2012). O óxi ou oxidado é uma droga parecida com o crack, seu principio ativo é a pasta base da folha de coca, porém no óxi são utilizados cal virgem e algum combustível (SILVA JUNIOR et al., 2012). Embora a utilização da cocaína se relacione menos com as lesões bucais pelo seu uso ser mais constante pela mucosa nasal, porém o seu uso na forma de crack pode ocasionar eritema, úlcera e hiperceratose na mucosa bucal, tão acentuada ou mais que as provocadas pelo uso do tabaco ou da maconha (WOYCEICHOSKI et al., 2007), além de erosão dental, semelhante à ocasionada pelo uso de metanfetaminas (BASSIOUNY et al., 2013). Não há na literatura, estudos avaliando o uso de óxi e merla e danos à saúde bucal. Embora, $40 \%$ dos pacientes avaliados fizessem uso de cocaína ou de seus derivados, hiperceratose e erosão dentária não foram achados frequentes. Isto pode ser explicado, pois alguns pacientes já não faziam uso da droga de abuso há algum tempo.

A maconha (Cannabis sativa) é uma droga perturbadora da atividade mental. Suas folhas e inflorescências secas podem ser fumadas ou ingeridas (MALONEY, 2011). Há diversos efeitos sistêmicos e manifestações orais associados ao uso de maconha (MALONEY, 2011), entre eles uvulite, estomatite nicotínica, aumento gengival semelhante ao induzido por fenitóína (RAWAL et al, 2012), perda óssea na periodontite (NOGUEIRA-FILHO et al., 2011) e diminuição da produção da saliva e consequente aumento do número de cáries de superfície (SCHULZ-KATTERBACH et al., 2009). Maconha foi a terceira droga mais utilizada pelos pacientes avaliados neste estudo (48\%), um fator importante para 
justificar a hipossalivação e alto índice CPOD nestes pacientes.

As substâncias mais utilizadas como calmantes hoje em dia são benzodiazepínicos, como por exemplo, o diazepam, bromazepam, lorazepam entre outros (DE ALMEIDA et al., 2008). Dependentes crônicos em sedativos e antidepressivos podem apresentar xerostomia aumentando a probabilidade do desenvolvimento cáries dentais (THOMSON et al., 2000; ALMEIDA et al., 2008; VISSINK et al., 2012). Neste estudo, a maioria dos pacientes (93\%) fazia uso de medicamento contínuo, sendo os principais antidepressivos, antiepiléticos, ansiolíticos, sedativos, antipsicóticos e tranquilizantes, todos estes relacionados ao tratamento para o abuso de drogas. Este é outro fator que pode ter contribuído para a hipossalivação observada nos pacientes assim como o alto índice de cárie nestes pacientes. Tal dado mostra a importância do questionamento quanto ao uso de medicamentos de uso contínuo, o qual pode estar relacionado à doença bucal dos pacientes.

É difícil relacionar determinada alteração bucal encontrada nos pacientes avaliados com uma droga específica, pois a maioria faz uso concomitante de mais de um tipo de droga, ou está em tratamento com medicamentos que também podem causar alterações bucais, conforme descrito anteriormente. Além disso, o uso de vários tipos de drogas simultaneamente poderia potencializar o aparecimento de lesões bucais, embora isto seja controverso na literatura (O'SULLIVAN, 2012).

Embora a maioria dos pacientes pesquisados tenha ido alguma vez ao dentista (98\%) e a última consulta odontológica tenha sido entre 2 a 3 anos (37\%), 90\% acreditavam que necessitava de algum tratamento, principalmente necessidade de prótese, extração, restauração e avaliação de fratura dentária. A necessidade de uso ou troca de prótese pela maioria dos pacientes (70\%) foi comprovada no exame bucal, assim como a necessidade de extração e restauração dentária, mostrando que estes pacientes tinham uma boa percepção de suas necessidades, embora sua saúde bucal não fosse boa. Estes achados concordam com os achados de O'Sullivan (2011), que também avaliou pacientes em Centros de reabilitação e observou alta taxa de doenças bucais não tratadas e somente tratamentos bucais emergenciais.

Com base nos dados obtidos, concluise que maioria dos pacientes usuários e exusuários apresentam várias alterações bucais associadas ao uso de drogas de abuso, tais como hipossalivação, $\mathrm{pH}$ ácido bucal, 
pigmentação exógena, além de saúde bucal muito comprometida.

\section{CONFLITO DE INTERESSE}

Os autores declaram não haver qualquer potencial conflito de interesse que possa interferir na imparcialidade deste trabalho científico.

\section{REFERÊNCIAS}

Ahmadi-Motamayel F, Goodarzi MT, Hendi SS, Kasraei S, Moghimbeigi A. Total antioxidant capacity of saliva and dental caries. Med Oral Patol Oral Cir Bucal. 2013; 18(4): e553-6. doi:10.4317/medoral.18762.

Barros MA, Pillon SC. Programa saúde da família: Desafios e potencialidades frente ao uso de drogas. Revista eletrônica de enfermagem. 2006; 8: 144-9.

Bassiouny MA. Dental erosion due to abuse of illicit drugs and acidic carbonated beverages. Gen Dent. 2013; 61(2):38-44.

Brand HS, Dun SN, Nieuw Amerongen AV. Ecstasy (MDMA) and oral health. British Dental Journal 2008; 204: 77-81. http://dx.doi.org/10.1038/bdj.2008.4

Brasil. Ministério da Saúde. Instituto Nacional de Câncer. 2006 [citado em 2013 Mar 15]. Disponível em: http://www.inca.gov.br/tabagismo/frameset. asp?item $=31$ maio2006\&link=produtos.$h t m$

Brasil. Ministério da Saúde. Portaria n³36/GM, de 19 de fevereiro de 2002. 2002. [citado em 2013 Mar 15]. Disponível em: http://portal.saude.gov.br/portal/arquivos/p df/Portaria\%20GM\%20336-2002.pdf .

Brasil. Presidência da República. LEI $\mathrm{N}^{\circ}$ 10.216, DE 6 DE ABRIL DE 2001. 2001. [citado em 2013 Mar 15]. Disponível em: http://www.planalto.gov.br/ccivil_03/leis/lei s_2001/l10216.htm

Bretas LP, Rocha ME, Vieira MS, Rodrigues ACP. Fluxo salivar e capacidade tamponante da saliva como indicadores de susceptibilidade á doença cárie. Rev Pesq Bras Odontoped Clin Integr. 2008; 8: 289-93. http://dx.doi.org/10.4034/1519.0501.2008.008 3.0006

Colodel EV, Silva ELFM, Zielak JC, Zaitter W, Crosato EM, Pizzatto E. Alterações bucais presentes em dependentes químicos. Rev Sul-Brasileira de Odontologia. 2008; 6: 44-8.

da Silva Junior RC, Gomes CS, Goulart Júnior SS, Almeida FV, Grobério TS, Braga JW, Zacca JJ, Vieira ML, Botelho ED, Maldaner AO. Demystifying "oxi" cocaine: Chemical profiling analysis of a "new Brazilian drug" from Acre State. Forensic Sci Int. 2012; 221(13):113-9. doi: 10.1016/j.forsciint.2012.04.015

D'Amore MM, Cheng DM, Kressin NR, Jones J, Samet JH, Winter M, Kim TW, Saitz R. Oral health of substance-dependent individuals: impact of specific substances. J Subst Abuse Treat. 2011; 41(2):179-85. doi: 10.1016/j.jsat.2011.02.005.

de Almeida Pdel V, Grégio AM, Brancher JA, Ignácio SA, Machado MA, de Lima $A A$, Azevedo LR. Effects of antidepressants and benzodiazepines on stimulated salivary flow rate and biochemistry composition of the saliva. Oral Surg Oral Med Oral Pathol Oral Radiol Endod. 2008;106(1):58-65. doi: 10.1016/j.tripleo.2007.11.008.

Fernandes JP, Brandão VSG, Lima AAS. Prevalência de lesões cancerizáveis bucais em indivíduos portadores de alcoolismo. Rev Brasileira de Cancerologia. 2008; 54: 239-44.

Gupta T, Shah N, Mathur VP, Dhawan A. Oral health status of a group of illicit drug users in 
Delhi, India. Community Dent Health. 2012;29(1):49-54.

Hamamoto DT, Rhodus NL. Methamphetamine abuse and dentistry. Oral Dis. 2009;15(1):27-37. doi: 10.1111/j.16010825.2008.01459.x.

Heng CK, Badner VM, Schiop LA. Meth mouth. N Y State Dent J. 2008;74(5):50-1.

Lopes CF, de Angelis BB, Prudente HM, de Souza BV, Cardoso SV, de Azambuja Ribeiro RI. Concomitant consumption of marijuana, alcohol and tobacco in oral squamous cell carcinoma development and progression: recent advances and challenges. Arch Oral Biol. 2012;57(8):1026-33. doi: 10.1016/j.archoralbio.2012.05.006.

Maloney WJ. Significance of cannabis use to dental practice. $\mathrm{N} Y$ State Dent J. 2011;77(3):36-9.

Maloney WJ. The significance of cocaine use to dental practice. $\mathrm{N} Y$ State Dent J. 2010;76(6):36-9.

Naidoo S, Smit D. Methamphetamine abuse: a review of the literature and case report in a young male. SADJ. 2011;66(3):124-7.

Nederfors T. Xerostomia and hyposalivation. Adv Dent Res. 2000;14:48-56. http://dx.doi.org/10.1177/08959374000140010 $\underline{701}$

Nogueira-Filho GR, Todescan S, Shah A, Rosa BT, Tunes Uda R, Cesar Neto JB. Impact of cannabis sativa (marijuana) smoke on alveolar bone loss: a histometric study in rats. J Periodontol. 2011;82(11):1602-7. doi: 10.1902/jop.2011.100362.

Oliveira D. Tragada Mortal. Rev Saúde São Paulo 2009; 15: 20-23.

O'Sullivan EM. Dental health of Irish alcohol/drug abuse treatment centre residents. Community Dent Health. 2012;29(4):263-7.

O'Sullivan EM. Prevalence of oral mucosal abnormalities in addiction treatment centre residents in Southern Ireland. Oral Oncol. 2011;47(5):395-9. doi: 10.1016/j.oraloncology.2011.03.003.

Péret ACA, Bonato KB. A participação do dentista na equipe multidisciplinar para o tratamento do paciente alcoolista. Rev Arqui Bras Odontol. 2008; 4:70-5.

Pillon SC, Martins M C. A relação entre a iniciação do uso de drogas e o primeiro ato infracional entre os adolescentes em conflito com a lei. Cadernos de Saúde Pública. 2008; 24

(5):1112-20.

http://dx.doi.org/10.1590/S0102$\underline{311 \times 2008000500018}$

Ravenel MC, Salinas CF, Marlow NM, Slate $\mathrm{EH}$, Evans ZP, Miller PM. Methamphetamine abuse and oral health: a pilot study of "meth mouth". Quintessence Int. 2012;43(3):22937.

Rawal SY, Tatakis DN, Tipton DA. Periodontal and oral manifestations of marijuana use. J Tenn Dent Assoc. 2012;92(2):26-31; quiz 312.

Reis SRA, Sadigursky $M$, Andrade MGS, Soares LP, Santo ARE, Boas DSV. Efeito genotóxico do etanol em células da mucosa bucal. Rev Pesquisa Odontológica Brasileira. 2002; 16 : 221-5. http://dx.doi.org/10.1590/S151774912002000300007

Ridenour TA, Bray BC, Cottler LB. Reliability of Use, Abuse, and Dependence of Four Types of Inhalants in Adolescents and Young Adults. Drug Alcohol Depend. 2007; 91(1): 40-9.

http://dx.doi.org/10.1016/j.drugalcdep.2007.05. 004 
Rosa EF, Inoue G, Takano RK, Assirati PFB, Corraini P, Carvalho VF et al. O papel do cirurgião dentista no abandono do hábito do fumo. R. Periodontia. 2009;19:68-74.

Schulz-Katterbach $\mathrm{M}$, Imfeld $\mathrm{T}$, Imfeld $\mathrm{C}$. Cannabis and caries - does regular cannabis use increase the risk of caries in cigarette smokers? Schweiz Monatsschr Zahnmed. 2009;119(6):576-83.

Serratine ACP, Silva MRM. Validação de um método simplificado de avaliação do $\mathrm{pH}$ salivar em crianças. Rev Pesq Bras Odontoped Clin Integr. 2009; 9:217-21. http://dx.doi.org/10.4034/1519.0501.2009.009 $\underline{2.0014}$

Silva LFG, Lopes FF, Oliveira AEF. Estudo sobre o fluxo salivar e xerostomia em mulheres na pré e pós-menopausa. Rev Pesq Bras Odontoped Clin Integr. João Pessoa. 2007; 7: 125-9.

Stead LF, Perera R, Bullen C, Mant D, Lancaster T. Nicotine replacement therapy for smoking cessation. Cochrane Database Syst Rev. 2008; 23(1):CD000146. doi: 10.1002/14651858.CD000146.pub3.

Thomson WM, Chalmers JM, Spencer AJ, Slade GD. Medication and dry mouth: findings from a cohort study of older people. J Public Health Dent. 2000;60(1):12-20. http://dx.doi.org/10.1111/j.17527325.2000.tb03286.x

Thomson WM, Poulton R, Broadbent JM, AlKubaisY S. Xerostomia and medications among 32-year-olds. Acta Odontol Scand. 2006; 64(4): 249-54. http://dx.doi.org/10.1080/00016350600633243

Traebert J, Moreira EAM. Transtornos alimentares de ordem comportamental e seus efeitos sobre a saúde bucal na adolescência. Rev Pesqui Odontol Bras. 2001; 15: 359-63. http://dx.doi.org/10.1590/S1517$\underline{74912001000400015}$
Vissink A, Visser A, Spijkervet FK. Oral medicine 1. Causes and clinical symptoms of dry mouth. Ned Tijdschr Tandheelkd. 2012;119(10):493-8.

http://dx.doi.org/10.5177/ntvt.2012.10.11249

Waszkiewicz N, Zalewska A, Szajda SD, Szulc A, Kępka A, Minarowska A, WojewódzkaŻelezniakowicz $M$, Konarzewska $B$, Chojnowska S, Supronowicz ZB, Ladny JR, Zwierz K. The effect of chronic alcohol intoxication and smoking on the activity of oral peroxidase. Folia Histochem Cytobiol. 2012;50(3):450-5. doi: 10.5603/19756.

Waszkiewicz N, Zalewska A, Szajda SD, Waszkiewicz $M$, Szulc A, Kepka A, Konarzewska B, Minarowska A, ZalewskaSzajda B, Wilamowska D, Waszkiel D, Ladny $J R$, Zwierz K. The effect of chronic alcohol intoxication and smoking on the output of salivary immunoglobulin A. Folia Histochem Cytobiol. 2012;50(4):605-8. doi: 10.5603/19709.

Waszkiewicz N, Zalewska A, Szulc A, Kepka A, Konarzewska B, Zalewska-Szajda B, Chojnowska S, Waszkiel D, Zwierz K. The influence of alcohol on the oral cavity, salivary glands and saliva. Pol Merkur Lekarski. 2011;30(175):69-74.

Woyceichoski IE, de Arruda EP, Resende LG, Machado MA, Grégio AM, Azevedo LR, de Lima AA. Cytomorphometric analysis of crack cocaine effects on the oral mucosa. Oral Surg Oral Med Oral Pathol Oral Radiol Endod. 2008;105(6):745-9. doi: 10.1016/j.tripleo.2007.09.011.

Recebido para publicação em 29/06/2013 Revisado em 29/11/2013

Aceito em 02/12/2013 